Rev. Int. Contam. Ambie. 35 (4) 807-814, 2019

DOI: $10.20937 /$ RICA.2019.35.04.03

\title{
MERCURIO EN RAÍCES AÉREAS Y ABSORBENTES DE Rhizophora mangle L. LOCALIZADA EN EL LITORAL COSTERO DE LA PROVINCIA DE EL ORO, ECUADOR
}

\author{
Mercury in aerial and absorbing roots in Rhizophora mangle L. located in the coast of El Oro province, Ecuador
}

\author{
Estefanía MOLINA ${ }^{1}$, Alfonso MARÍN MEDINA ${ }^{1}$, Byron LAPO CALDERÓN ${ }^{1 *}$, \\ Víctor Hugo GONZÁLEZ ${ }^{1}$ y Mairin LEMUS ${ }^{2,3}$
}

\author{
${ }^{1}$ Universidad Técnica de Machala, Av. Panamericana, km 5 1/2 Vía a Pasaje, Machala, C.P. 070102, Ecuador \\ ${ }^{2}$ Universidad de Oriente, Cumaná, Estado Sucre, C.P. 6101, Venezuela \\ ${ }^{3}$ Secretaría Nacional de Ciencia y Tecnología, Programa Prometeo, Whymper E7-37, Quito, C.P. 170516, \\ Ecuador \\ *Autor para correspondencia: byronglc@gmail.com
}

(Recibido: septiembre 2017; aceptado: diciembre 2018)

Palabras clave: bioacumulación, contaminación, metal pesado, manglares

\section{RESUMEN}

Se evaluaron las concentraciones de Hg en raíces absorbentes y aéreas de Rhizophora mangle L., en cuatro zonas costeras de la provincia de El Oro, Ecuador, correspondientes a Puerto Hualtaco, Estero Huaylá, Jambelí y Bajo Alto durante los meses de noviembre de 2014 (periodo de poca lluvia) y enero de 2015 (periodo de lluvia). Se recolectaron un total de 80 raíces, 10 aéreas y 10 absorbentes en cada zona. El Hg se determinó mediante espectrofotometría de absorción atómica acoplado a generación de vapor de hidruros en frío y expresado en $\mathrm{mg} / \mathrm{kg}$ (materia seca). La concentración de $\mathrm{Hg}$ en las raíces aéreas y absorbentes no presentó diferencias significativas, con valores de $243.13 \pm 91.14$ y $300.03 \pm 170.96 \mathrm{mg} / \mathrm{kg}$, respectivamente. Las raíces absorbentes presentaron una concentración de $\mathrm{Hg}$ significativamente más elevada durante la época de lluvia, con un valor de $321.68 \pm 85.59 \mathrm{mg} / \mathrm{kg}$. La localidad de Puerto Hualtaco presentó el mayor valor de $\mathrm{Hg}(382.46 \pm 273.84 \mathrm{mg} / \mathrm{kg})$ en las raíces absorbentes. Estos hallazgos demuestran que $R$. mangle L. tiene una gran capacidad de incorporación de $\mathrm{Hg}$ y puede ser un buen biomonitor de mercurio en los ecosistemas de manglares.

Key words: bioaccumulation, contamination, heavy metal, mangroves

\begin{abstract}
Mercury concentrations in absorbent and aerial roots of Rhizophora mangle L. were investigated in the coastline of El Oro province, Ecuador. Sampling of roots was conducted in Puerto Hualtaco, Estero Huaylá, Jambelí and Bajo Alto during the months of November 2014 (dry seasons) and January 2015 (rainy seasons). Eighty roots were collected, 10 aerial and 10 absorbent in each location. Mercury was determined using atomic absorption spectrophotometry coupled to hydride-cold vapor generation and was expressed as $\mathrm{mg} / \mathrm{kg}$ of dry mass. The highest concentrations of $\mathrm{Hg}$ were observed during the raining season, with a value of $321.68 \pm 85.59 \mathrm{mg} / \mathrm{kg}$. The location of Puerto Hualtaco presented the highest value of $\mathrm{Hg}(382.46 \pm 273.84 \mathrm{mg} / \mathrm{kg})$ in absorbent
\end{abstract}


roots. The results showed that $\mathrm{Hg}$ concentration in samples of aerial and absorbing roots are characteristic of an area highly impacted by $\mathrm{Hg}$. This findings demonstrate that $R$. mangle $\mathrm{L}$. has a great capacity to incorporate $\mathrm{Hg}$ in its roots and can be a good biomonitor of mercury in mangrove ecosystems.

\section{INTRODUCCIÓN}

Los manglares constituyen uno de los ecosistemas más productivos del mundo y tienen un gran valor ecológico y económico, debido a que proveen sitios de refugio, crianza y reproducción de la diversa fauna que habita en el mangle. A su vez, las raíces zancudas, los detritos de las hojas de los árboles del mangle, así como los compuestos orgánicos solubles, proveen nutrimentos y sustrato para muchos organismos como bivalvos, cangrejos y varias especies de peces. Por otro lado, los ecosistemas del mangle actúan como sumideros naturales, y sirven como filtros para sedimentos y nutrimentos, manteniendo la calidad del agua. Asimismo, representan barreras físicas y biogeoquímicas para contaminantes metálicos y sirven como amortiguadores de inundaciones (Nagelkerken et al. 2008, Zhou et al. 2011).

Los mangles tienen la capacidad de absorber metales pesados; sin embargo, concentraciones elevadas de estos elementos en sedimentos pueden afectar su eficiencia de incorporación. Adicionalmente, las raíces, hojas y frutos se caen y forman parte de la materia orgánica, que a su vez sirve de alimento a la comunidad bentónica e inclusive como nutriente para los manglares, lo cual propicia recirculación, bioacumulación y biomagnificación de estos elementos (MacFarlane et al. 2007, Wang'ondu et al. 2014).

El mercurio $(\mathrm{Hg})$ es el elemento que representa mayor preocupación a nivel mundial debido a su acción tóxica para los organismos y para el hombre. Habitualmente no se encuentra en el medio natural y su presencia significa una contaminación de carácter antrópico. Según la Agencia para la Protección Ambiental de los Estados Unidos (US-EPA, por sus siglas en inglés), el Hg es unos de los elementos más peligrosos, y es perjudicial aún en pequeñas concentraciones (Posada y Arroyave 2006, Thangaradjou et al. 2014).

Dentro de las fuentes de contaminación que generan $\mathrm{Hg}$ y descargan directamente sus vertidos hacia los cuerpos hídricos se encuentran las industrias minera, petroquímica, cementera, agroquímica, cartonera, electroquímica, de pinturas y de pilas, además de los vertidos domésticos. Una vez descargado en los cuerpos de agua y depositado en los sedimentos, se inicia la biotransformación del $\mathrm{Hg}$ a una de sus especies químicas más tóxicas, el metilmercurio, que luego es incorporado a la cadena trófica (Sanders et al. 2008). Esta contaminación ocasiona que todas las descargas que se realizan a los cuerpos de agua, tengan como destino final las zonas costeras, lo cual ha representado un problema de salud para los ecosistemas.

Las directrices canadienses sobre la calidad de sedimentos para la protección de la vida acuática (ISQG, por sus siglas en inglés) establecen una concentracion de $\mathrm{Hg}$ no superior a $0.17 \mathrm{mg} / \mathrm{kg}$, mientras que el nivel de efecto probable (PEL), parámetro que define la concentración sobre la cual podrían ocurrir efectos biológicos adversos, es superior a $0.486 \mathrm{mg} /$ $\mathrm{kg}$ (CCME 2001): por lo nterior, se puede tener una idea clara respecto dl problema de contaminación por $\mathrm{Hg}$ y los daños que produce en muchos ecosistemas acuáticos.

En el Ecuador existen leyes e instituciones gubernamentales encargadas de proteger los manglares. Al respecto los artículos 405 y 406 del Sistema Nacional de Áreas Protegidas establecen que se garantizará la conservación de la biodiversidad y el mantenimiento de las funciones ecológicas; asimimso, que el Estado regulará la conservación, manejo y uso sostenible, recuperación y limitaciones de dominio de los ecosistemas frágiles y amenazados (SENPLADES 2013).

Actualmente hay organizaciones encargadas de la protección de los manglares en el Ecuador, entre las cuales se encuentran la Corporación Coordinadora Nacional para la Defensa del Ecosistema Manglar (C-CONDEM), que otorga las tierras a los Pueblos Ancestrales del Ecosistema de Manglares y reclama el derecho a la propiedad colectiva de las zonas de manglares ecuatorianos (Latorre y Farrell 2014).

La provincia de El Oro debe su nombre a las elevadas reservas de ese metal que se encuentran en ella, que es uno de los recursos más explotados de la región desde hace más de 100 años. La actividad minera es una de las principales fuentes de contaminación de la zona, ya que durante muchos años los 
efluentes de dicha actividad se han descargado en forma directa a los cursos de agua. Éstos son utilizadas luego para riego, produciéndose así un ciclo de contaminación.

Una de las mayores zonas mineras del Ecuador se encuentra en la cuenca alta del Portovelo-Zaruma, donde opera gran cantidad pequeñas emepresas, la mayoría de ellas pequeñas, que utilizan el amalgamiento con $\mathrm{Hg}$ como forma de extracción de oro. Aproximadamente 1.5 t/año de HG son liberadas en Portovelo-Zaruma, de las cuales se evapora el $70 \%$ y el $30 \%$ se libera con relaves (Velásquez-López et al. 2010).

La minería ha ocasionado una severa pérdida de biodiversidad y una significativa incorporación de metales, particularmente de $\mathrm{Hg}$, en la biota, no sólo por sus descargas a los cuerpos de agua sino por la elevada concentración de $\mathrm{Hg}$ atmosférico durante la quema, que puede llegar hasta $193.8 \mathrm{mg} / \mathrm{m}^{3}$ (Sandoval 2001).

El objetivo del presente trabajo fue determinar las concentraciones de $\mathrm{Hg}$ en raíces aéreas y absorbentes de Rhizophora mangle L. localizada en zonas del litoral costero perteneciente a la provincia de El Oro en Ecuador.

\section{MATERIALES Y METODOS}

\section{Descripción del área de estudio}

La investigación se realizó en la zona costera de la provincia de El Oro, ubicada al suroeste del Ecuador, la cual cuenta con una extensión de aproximadamente $203 \mathrm{~km}$. Para ello se seleccionaron las localidades de Puerto Hualtaco, Estero Huaylá, Jambelí y Bajo Alto, cuyas coordenadass UTM Wgs $84 \mathrm{Z} 17 \mathrm{~S}$ fueron (585631; 9618421), (613027; 9639698), (608625; 9642159) у (622264; 9656755), respectivamente (Fig. 1).

\section{Campo}

Se tomaron secciones de raíces absorbentes y aérea de árboles maduros de $R$. mangle durante noviembre de 2014 (precipitación promedio: $3.6 \mathrm{~mm} / \mathrm{m}^{2}$ ), pertenecientes a la época de poca lluvia, y enero de 2015 (precipitación promedio: $37.0 \mathrm{~mm} / \mathrm{m}^{2}$ ), perteneciente a la época de lluvia. Las muestras se obtuvieron utilizando un bisturí con hojilla de acero inoxidable para cada muestra y posteriormente se colocaron en bolsas plásticas de cierre hermético previamente rotuladas, Luego se trasladaron al

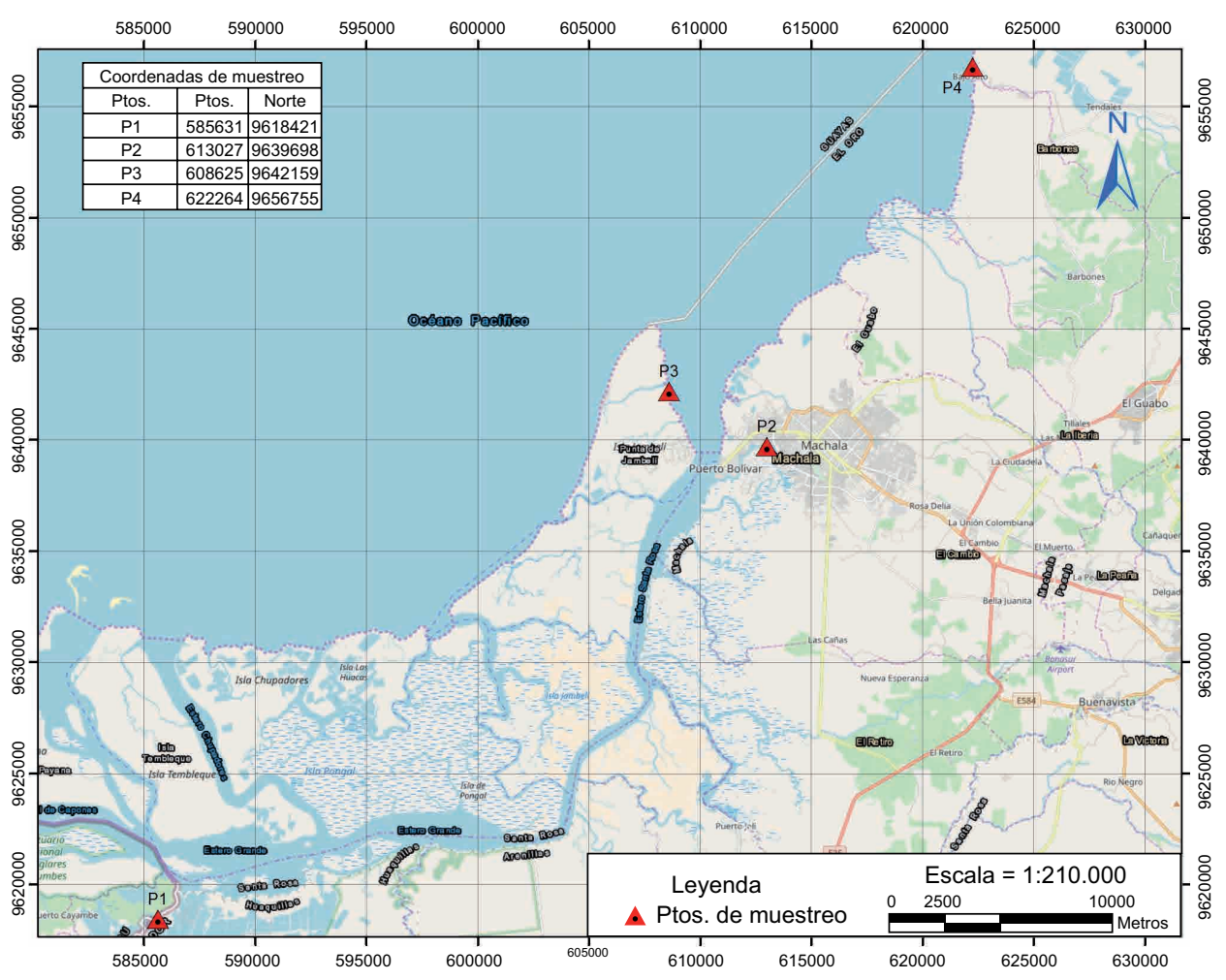

Fig. 1. Localidades de muestreo en la zona costera de la provincia de El Oro, Ecuador. P1: puerto Hualtaco, P2: Bajo Alto, P3: Estero Huaylá y P4: Jambelí 
laboratorio en una cava con hielo. Se recolectaron un total de 80 muestras de raíz (40/época, 10/localidades y $5 /$ cada tipo de raíz).

\section{Laboratorio}

El material vegetal fue molido hasta aproximadamente $8 \mu \mathrm{m}$ con un molino manual. Posteriormente se secó en una estufa Boeco a $60{ }^{\circ} \mathrm{C}$ durante 72 h. Una vez homogeneizada la muestra, se pesó un gramo de ellas y se colocó en un recipiente de PVC previamente lavado con $\mathrm{HNO}_{3}$ al $10 \%$. Las muestras se predigirieron con $7 \mathrm{~mL}$ de $\mathrm{HNO}_{3}$ concentrado grado analítico marca J. T. Barker a $65 \%$ durante $24 \mathrm{~h}$, luego se colocaron en baño maría durante $4 \mathrm{~h}$ a temperatura máxima de $60^{\circ} \mathrm{C}$ hasta completar la digestión. Transcurrido este tiempo se procedió a filtrarlas con papel filtro marca Macherey-Nagel Mn615 de $125 \mathrm{~mm}$ y se agregó agua desionizada a la muestra hasta completar $25 \mathrm{~mL}$, para luego añadir cinco gotas de permanganato de potasio (Peven y Uhler 1993).

Para determinar el $\mathrm{Hg}$ en las muestras biológicas se utilizó un espectrofotómetro de absorción atómica acoplado a generación de vapor de hidruros en frio, utilizando un equipo Shimadzu AA6300. La recuperación, eficiencia y precisión de la técnica empleada para la extracción de $\mathrm{Hg}$ se medió utilizando el material certificado de pesado (IAEA-407, International Atomic Energy Agency, Mónaco), para lo cual se procesaron tres muestras en las mismas condiciones de la curva de calibración y las muestras de tejidos de mangle. La concentración teórica de $\mathrm{Hg}$ es de 0.22 con un intervalo de 0.216-0.228 y la obtenida en este trabajo fue $0.21 \pm 0.019$ con un intervalo de 0.199 0.237 y una recuperación de $97 \%$.

\section{Análisis de datos}

Dado que los datos no cumplieron con los supuestos a priori de homocedasticidad y distribución normal, se procedió a utilizar los análisis no paramétricos. Para determinar las diferencias en las concentraciones de $\mathrm{Hg}$ en raíces absorbentes y aéreas de $R$. mangle se aplicó la prueba de Mann-Whitney (W), la cual también se efectuó para determinar las diferencias durante los dos periodos de muestreo. Para los niveles de $\mathrm{Hg}$ en las cuatro localidades se aplicó un análisis de varianza no paramétrico de Kruskal-Wallis (KW) y la prueba de la mediana de Mood, evaluándose la significancia al $5 \%(\mathrm{p}<0.05)$. Los análisis computarizados de toda la estadística aplicada se realizaron utilizando el paquete Statgraphics Centurion XVI, v. 16.1.15

\section{RESULTADOS Y DISCUSIÓN}

Los resultados obtenidos en la presente investigación demuestran una gran incorporación de $\mathrm{Hg}$ en raíces de $R$. mangle en la zona marino-costera de la provincia de El Oro, Ecuador, con un valor promedio de $\mathrm{Hg}$ en las raíces de $271.58 \pm 131.05 \mathrm{mg} / \mathrm{kg}$. Esto pudiera estar asociado con las descargas de los ríos provenientes de las cuencas altas, que tienen una elevada actividad minera industrial y artesanal, siendo esta última la que probablemente más ha contribuido al incremento de este metal en los ecosistemas acuáticos. Tarras-Wahlberg et al. 2001 señalaron concentraciones de $\mathrm{Hg}$ de 1 a $5 \mathrm{mg} / \mathrm{kg}$ en sedimentos del río Calera en la región Portovelo-Zaruma de El Oro, Ecuador, estimando descargas de $289 \mathrm{~kg} / \mathrm{año}$, mientras que Velásquez-López et al. 2010 estimaron que un $\mathrm{Hg}$ total liberado en esa misma región de $1467.05 \pm 115.76 \mathrm{~kg} /$ año.

Hay pocos estudios que reporten concentraciones de $\mathrm{Hg}$ en $R$. mangle similares a las del presente trabajo, y los realizados han señalado concentraciones más bajas de Hg: Marchand et al. (2006) estimaron un promedio de $0.11 \mathrm{mg} / \mathrm{kg}$ en mangles de las costas de la Guayana Francesa; Qiu et al. (2011) encontraron una concentración de $0.2 \mathrm{mg} / \mathrm{kg}$ en manglares de la isla Hainan, China, y Ding et al. (2010) detectaron valores de $1.76 \mathrm{mg} / \mathrm{kg}$ de $\mathrm{Hg}$ total y $0.72 \mathrm{mg} / \mathrm{kg}$ de metilmercurio en manglares de la zona costera de China (Dongzhaigang, Sanya, Shenzhen, Zhanjiang, Daguanshan y Fugong).

Hasta donde llega el conocimiento de los autores, si bien la literatura no muestra la incorporación de $\mathrm{Hg}$ en raíces de manglares con concentraciones elevadas, las plantas pueden concentrar el metal en este tejido en magnitudes muy superiores a las encontradas en el entorno, como ocurre con el esparrago Asparagus acutifolius, el cual presenta concentraciones extremadamente altas de $\mathrm{Hg}$ en raíces $(0.6 \mathrm{a} 443 \mu \mathrm{g} / \mathrm{g})$ y tallos $(0.3$ a $140 \mu \mathrm{g} / \mathrm{g})$ cuando crece en suelos con una concentración de $4220 \mu \mathrm{g} / \mathrm{g}$ (Martínez-Coronado et al. 2011), como la de este trabajo.

Los elevados niveles de $\mathrm{Hg}$ en la zona costera de El Oro están asociados principalmente con la incorporación de este elemento en los sedimentos. De acuerdo con estudios realizados por Marín et al. (2016), los niveles de $\mathrm{Hg}$ en la zona costera de la provincia de El Oro se encuentran en un rango de 3.39 a $8.86 \mathrm{mg} / \mathrm{kg}$.

El enriquecimiento en los sedimentos superficiales es probablemente consecuencia de las actividades industriales y domésticas que han caracterizado a la provincia de El Oro. Una de ellas y quizás la más 
importante está relacionada con las actividades de la minería que datan de 1895, aproximadamente cuando aparecieron las primeras empresas dedicadas a la explotación minera (Murillo 2000). Otra posible causa del enriquecimiento de $\mathrm{Hg}$ en los sedimentos está relacionada con el uso de agroquímicos (óxido de mercurio, cloruro de mercurio [calomel], acetato de fenilmercurio [PMA], oleato fenilmercúrico [PMO], mercurio alquilo, mercurio alcoxialquilo y arilo), los cuales fueron prohibidos desde noviembre de 2005 por la US-EPA, la Unión Europea y el Convenio de Rótterdam sobre el Consentimiento Fundamentado Previo (RAS 2011), Una tercera fuente son las descargas residuales sin tratamiento previo, que presumiblemente se han realizando a lo largo de los años debido al el desarrollo urbanístico e industrial orense.

Las concentraciones de $\mathrm{Hg}$ en las raíces aéreas y absorbentes se muestran en la Figura 2. En promedio, se encontró una concentración de $300.03 \pm 170.96$ $\mathrm{mg} / \mathrm{kg}$ para raíces absorbentes, mientras que en las raíces aéreas fue de $243.13 \pm 91.14 \mathrm{mg} / \mathrm{kg}$. La prueba de Mann-Whitney ( $\mathrm{W}=639.0 ; \mathrm{p}=0.12$ ) no estableció diferencias estadísticamente significativas en las concentraciones de $\mathrm{Hg}$ entre los dos tipos de raíces.

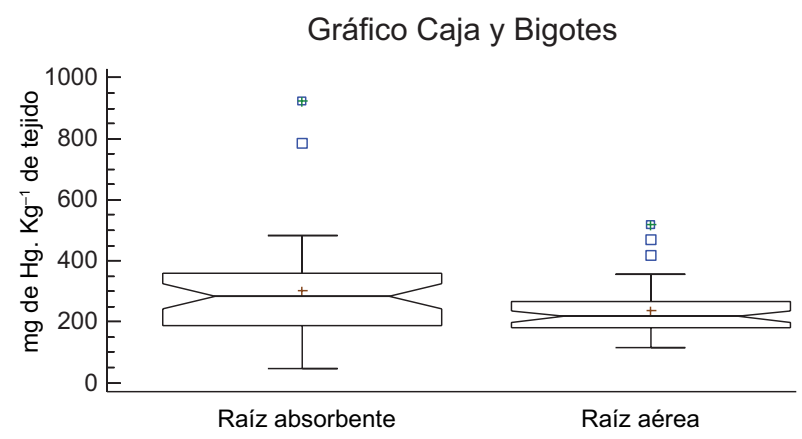

Fig. 2. Concentración promedio de $\mathrm{Hg}$ ( $\mathrm{mg} / \mathrm{kg}$ en masa seca) en dos tipos de raíces de Rhizophora mangle L. en la zona costera de la provincia de El Oro, Ecuador. La línea vertical representa el rango de valores; los puntos superiores, valores atípicos; la línea media dentro de la caja, la mediana, y el signo de + , la localización de la media

La compartimentación de $\mathrm{Hg}$ en los diferentes órganos de la planta depende de los mecanismos de translocación del metal (Moreno-Jiménez et al. 2006), pudiendo en muchos casos ser una estrategia de las plantas para acumular más Hg en las raíces que en las estructuras aéreas, como las hojas y los frutos
(Dago et al. 2014). No obstante, la incorporación de $\mathrm{Hg}$ en los órganos aéreos no sólo depende de la movilización del metal absorbido por las raíces de sedimentos enriquecidos con $\mathrm{Hg}$, sino también de la incorporación del $\mathrm{Hg}$ que se encuentra en la atmosfera, ya que el tejido foliar es capaz de incorporar y liberar fácilmente $\mathrm{Hg}$ atmosférico (Ericksen et al. 2003). Esto podría ocurrir en las raíces aéreas, las cuales presentan varias vías de incorporación de $\mathrm{Hg}$; además, la liberación del mismo puede estar asociada a cambios en los factores ambientales, particularmente de temperatura, lo que establece un mecanismo de incorporación y liberación más dinámico que las raíces absorbentes.

El mangle se ha desarrollado a lo largo de la evolución creando una serie de mecanismos a nivel celular que le permiten establecerse en ambientes con altas concentraciones de metales. Con la participación de microorganismos rizosféricos, las plantas forman placas de hierro que permiten la adsorción de metales, quedando total o parcialmente inmovilizados. En la absorción a nivel celular, la planta produce exudados radicales, metalotioneínas y fitoquelatinas que participan en el enlazamiento y metabolización de los metales (González-Mendoza et al. 2008).

Al parecer, los mangles tienen una gran tolerancia a la contaminación por $\mathrm{Hg}$, pues se ha demostrado que las plántulas de mangle rojo ( $R$. mangle) tratados con dosis de 10 a $500 \mu \mathrm{g} / \mathrm{g}$ de metal en suelo no se observan seriamente afectadas. Sólo resultan afectadas cuando la dosis de exposición es superior a 500 $\mu \mathrm{g} / \mathrm{g}$ en suelo. Los autores atribuyen la tolerancia de las plántulas de $R$. mangle a altas concentraciones del metal a la formación de sulfitos no tóxicos en el interior de la raíz o en su superficie, a un mecanismo de desintoxicación en los tejidos de exclusión iónica de las raíces o a una combinación de ambos factores (Walsh et al. 1979).

Los valores encontrados en las raíces absorbentes y raíces aéreas en época de lluvias fueron aproximadamente un 13 y $22 \%$ más altos, respectivamente, que en época de lluvias escasas.

Los valores encontrados en las raíces absorbentes en época de lluvias fueron aproximadamente un 13 $\%$ más altos que en época de lluvias, mientras que en las raíces aéreas fueron de $22 \%$ en la misma época. Es posible que los mayores enriquecimientos de los sedimentos en zonas costeras estén determi-nados por el aumento en la deposición de material particulado enriquecido con el $\mathrm{Hg}$ arrastrado durante las épocas de lluvias a través de las descargas fluviales y la precipitación atmosférica. 

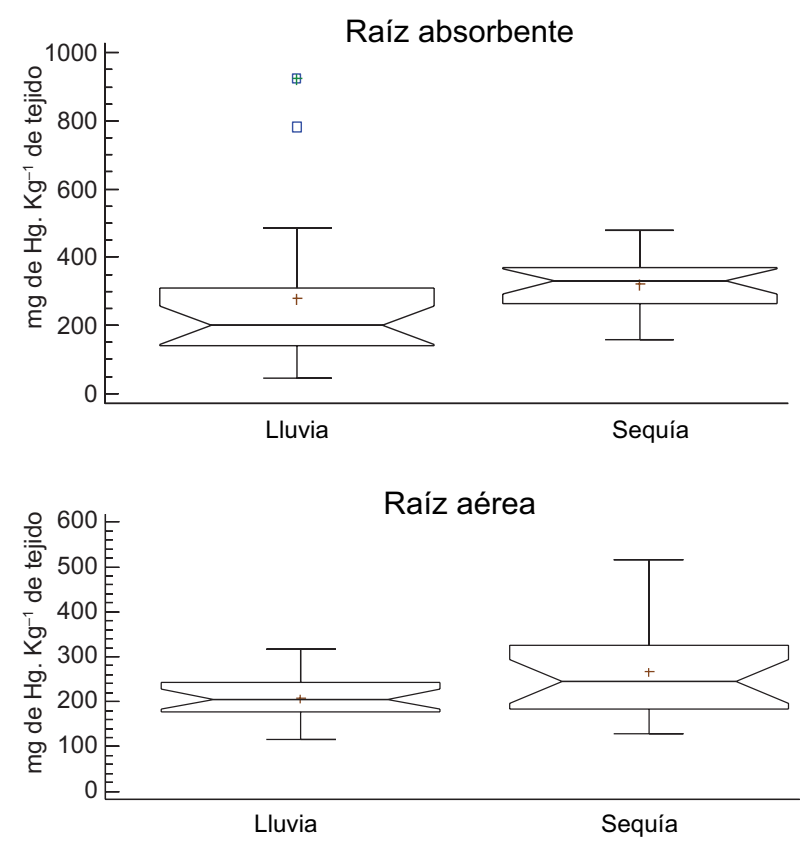

Fig. 3. Concentración de $\mathrm{Hg}(\mathrm{mg} / \mathrm{kg}$ en masa seca) en raíces absorbentes y aéreas de Rhizophora mangle L. en épocas de lluvia y de lluvia escasa en la zona costera de la provincia de El Oro, Ecuador. La línea vertical representa el rango de valores; los puntos superiores, valores atípicos; la línea media dentro de la caja, la mediana, y el signo de + , la localización de la media

En la zona de estudio, las precipitaciones comienzan en el mes de noviembre y los ríos descargan sus aguas en las zonas costeras, arrastrando consigo el Hg proveniente de la minería; allí, a su vez, el metal es movilizado por las corrientes marinas y depositado a lo largo de los ecosistemas costeros (Paredes et al. 2008).

En las raíces absorbentes no se obtuvieron diferencias significativas $(\mathrm{KW}=1.93 ; \mathrm{p}=0.585)$ con relación a las localidades; sin embargo, se observa un mayor rango de concentración en Puerto Hualtaco (45.70-921.99 mg/kg) (Fig. 4). En cuanto a las raíces aéreas, sí hubo diferencias significativas $(\mathrm{KW}=$ $18.10 ; p=0.0004)$. La prueba de medianas de Mood indicó que la máxima concentración de Hg ocurrió en la localidad del Estero Huaylá $(346.48 \pm 99.69 \mathrm{mg} / \mathrm{kg}$ ) y la menor en la localidad de Puerto Hualtaco (178.52 $\pm 34.53 \mathrm{mg} / \mathrm{kg}$ ).

Las raíces aéreas de mangles de Estero Huaylá presentaron concentraciones alrededor del $34 \%$ por encima del resto de las localidades. La incorporación del $\mathrm{Hg}$ en las raíces absorbentes proviene principalmente del $\mathrm{Hg}$ biodisponible en el sedimento y en segundo lugar del biodisponible en la columna de agua (Gustin et al. 2000), siendo este órgano un
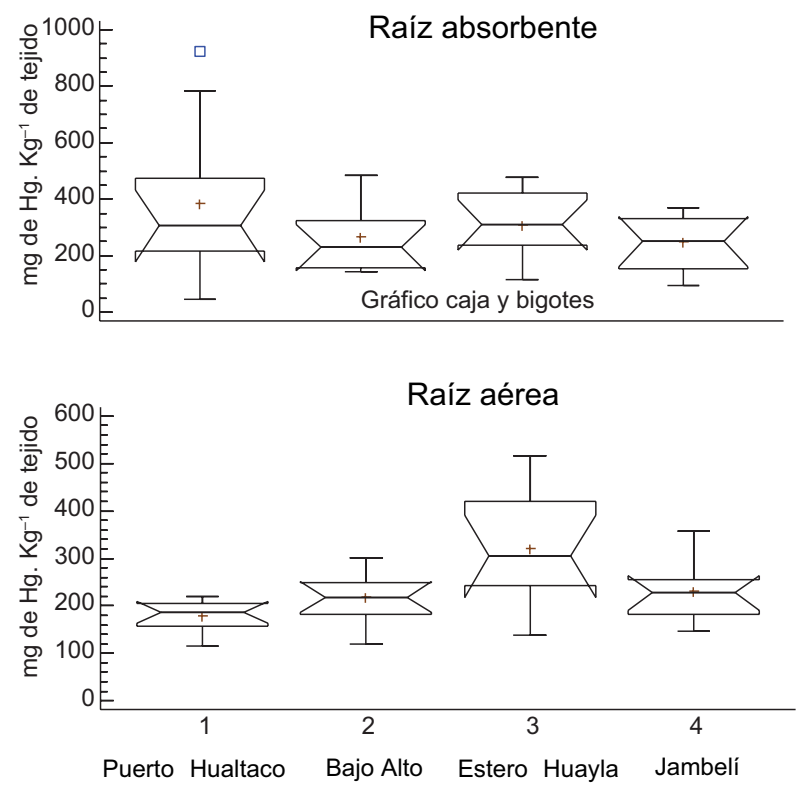

Fig. 4. Concentración de $\mathrm{Hg}(\mathrm{mg} / \mathrm{kg}$ en masa seca) en raíces absorbentes y aéreas de Rhizophora mangle L. en cuatro localidades de Puerto Hualtaco, Bajo Alto, Estero Huaylá y Jambelí en la zona costera de la provincia de El Oro, Ecuador. La línea vertical representa el rango de valores; los puntos superiores, valores atípicos; la línea media dentro de la caja, la mediana, y el signo de + , la localización de la media

bioindicador de $\mathrm{Hg}$ en cuerpos de agua contaminados, mientras que las raíces aéreas tienen al menos tres vías de incorporación del metal: 1) la traslocación del Hg incorporado a través de las raíces absorbentes (Zhang et al. 2012), 2) la bioacumulación directamente de la columna de agua durante la pleamar y 3) la deposición atmosférica (Scholtz et al. 2003). En el caso de Puerto Hualtaco, éste recibe las aguas de las principales cuencas mineras de la provincia de El Oro y el río internacional Zarumilla, que divide la frontera de Ecuador y Perú, mientras que la localidad de Estero Huaylá se caracteriza por estar localizada en un estero que se utiliza como descarga de las aguas residuales del sur de la ciudad donde no desembocan ríos provenientes de zonas mineras, por lo que posiblemente la deposición atmosférica y las mareas desempeñen un papel importante en los elevados niveles de $\mathrm{Hg}$ que presentaron las raíces aéreas.

Los datos registrados indicaron que el área de estudio presenta una intervención antrópica importante con elevada incorporación de $\mathrm{Hg}$, lo que pudiera representar un peligro ambiental potencial para la salud de los ecosistemas de manglar, la vida silvestre y los habitantes de la Provincia de El Oro. Por ello se requiere una acción inmediata a través de procedimientos de remediación. 


\section{CONCLUSIONES}

Luego de la evaluación de la concentración de $\mathrm{Hg}$ en raíces aéreas y absorbentes de mangle (Rhizophora mangle L.), se determinó que existen elevadas concentraciones de este metal pesado en todas las muestras evaluadas. Éstas comprendieron raíces aéreas y absorbentes recolectadas en cuatro puntos de muestreo y dos estaciones climáticas: seca y lluviosa. La mayor concentración fue detectada en las raíces absorbentes en época de lluvias. Por otra parte, no se encontraron diferencias significativas en la concentración de $\mathrm{Hg}$ en los diferentes puntos de muestreo, aunque sí se evidenciaron diferencias entre los periodos seco y lluvioso.

\section{REFERENCIAS}

CCME (2001). Canadian sediment quality guidelines for the protection of aquatic life, summary tables. En: Canadian environment quality guidelines. Canadian Council of Ministers of the Environment, Winnipeg, Canadá, 5 pp.

Dago A., González I., Ariño C., Martínez-Coronado A., Higueras P., Díaz-Cruz J.M. y Esteban M. (2014). Evaluation of mercury stress in plants from the Almadén mining district by analysis of phytochelatins and their Hg complexes. Environ. Sci. Technol. 48 (11), 62566263. DOI: $10.1021 / \mathrm{es} 405619 \mathrm{y}$

Ding Z.H., Liu J.L., Li L.Q., Lin H.N., Wu H. y Hu Z.Z. (2010). Distribution of $\mathrm{Hg}$ in mangrove plants and correlation with $\mathrm{Hg}$ speciation in sediments. Environ. Sci. 31 (9), 2234-2239.

Ericksen J.A., Gustin M.S., Schorran D.E., Johnson D.W., Lindberg S.E. y Coleman J.S. (2003). Accumulation of atmospheric mercury in forest foliage. Atmos. Environ. 37 (12), 1613-1622.

DOI: $10.1016 / \mathrm{S} 1352-2310(03) 00008-6$

González-Mendoza D., Gimaldo O. y Cervantes L. (2008). Los elementos potencialmente tóxicos en las plantas de manglar: una revisión de los mecanismos de tolerancia involucrados. Rev. Cien. Tec. América 33 (11), 817-820.

Gustin M., Biester H. y Kim C. (2002). Investigation of the light-enhanced emission of mercury from naturally enriched substrates. Atmos. Environ. 36 (20), 32413254. DOI: $10.1016 / \mathrm{S} 1352-2310(02) 00329-1$

Latorre S. y Farrell K. (2014). The disruption of ancestral peoples in Ecuador's mangrove ecosystem: Class and ethnic differentiation within a changing political context. Latin American and Caribbean Ethnic Studies 9 (3), 293-317. DOI: 10.1080/17442222.2014.959777
MacFarlane G., Koller C. y Blomberg S. (2007). Accumulation and partitioning of heavy metals in mangroves: A synthesis of field-based studies. Chemosphere 69 (9), 1454-1464. DOI: 10.1016/j.chemosphere.2007.04.059

Marchand C., Lallier-Vergès E., Baltzer F., Albéric P., Cossa D. y Baillif P. (2006). Heavy metals distribution in mangrove sediments along the mobile coastline of French Guiana. Mar. Chem. 98 (1), 1-17.

DOI: $10.1016 /$ j.marchem.2005.06.001

Marín A., González V., Lapo B., Molina E. y Lemus M. (2016). Niveles de mercurio en sedimentos superficiales en tres localidades de la provincia de El Oro. Gayana 8 (2), 147-153.

DOI: $10.4067 / \mathrm{S} 0717-65382016000200147$

Martínez-Coronado A., Oyarzún R., Esbrí J.M., Llanos W. e Higueras P. (2011). Sampling high to extremely high $\mathrm{Hg}$ concentrations at the Cerco de Almadenejos, Almadén mining district (Spain): The old metallurgical precinct (1794 to 1861 AD) and surrounding areas. J. Geochem Explor. 109 (1-3), 70-77.

DOI: 10.1016/j.gexplo.2010.04.007

Moreno-Jiménez E., Gamarra R., Carpena-Ruiz R.O., Millán R., Peñalosa J.M. y Esteban E. (2006). Mercury bioaccumulation and phytotoxicity in two wild plant species of Almadén area. Chemosphere 63 (11), 1969-1973.

DOI: 10.1016/j.chemosphere.2005.09.043

Murillo R. (2000). Zaruma, historia minera identidad en Portovelo. Abya-Yala, Quito, Ecuador, 181 pp.

Nagelkerken I., Blaber S., Bouillon S., Verde P., Haywood M., Kirton L., Meynecke J., Pawlik J., Penrose H., Sasekumar A. y Somerfield P. (2008). The habitat function of mangroves for terrestrial and marine fauna. Aquatic Botany 89, 155-185.

DOI: 10.1016/j.aquabot.2007.12.007

Paredes F., Millano J. y Guevara E. (2008). Análisis espacial de las sequías meteorológicas en la región de Los Llanos de Venezuela durante el período 1961-1996. Revista Climatología 8 (1), 15-27.

Peven C.S. y Uhler A.D. (1993). Analytical procedures for trace and major element analysis. En: Sampling and analytical methods of the national status and trends program national benthic surveillance and mussel watch project, vol. I (Lauenstein G.G. y Cantillo A.Y., Eds.). Silver Spring, Maryland, pp. 213-219.

Posada M. y Arroyave M. (2006). Efectos del Hg sobre algunas plantas acuáticas tropicales. Rev. EIA Esc. Ing. Antioq. 6, 57-67.

Qiu Y., Yu K., Zhang G. y Wang W. (2011). Accumulation and partitioning of seven trace metals in mangroves and sediment cores from three estuarine wetlands of Hainan Island, China. J. Hazard Mater. 190 (1-3), 631638. DOI: 10.1016/j.jhazmat.2011.03.091 
RAS (2011). Lista de plaguicidas prohibidos. Listado. Red de Agricultura Sostenible, San José, Costa Rica, 9 pp.

Sandoval F. (2001). La pequeña minería en el Ecuador. International Institute for Environment and Development, Londres, Inglaterra, $31 \mathrm{pp}$.

Sanders C., Santos E., Silva E. y Patchineelam S. (2008). Contrasting mercury and manganese deposition in a mangrove-dominated estuary (Guaratuba Bay, Brazil). Geo. Mar. Lett. 28 (4), 239-244.

DOI: $10.1007 / \mathrm{s} 00367-008-0104-8$

SENPLADES (2013). Plan Nacional Buen Vivir. Secretaría Nacional de Planificación y Desarrollo, Quito, Ecuador, 674 pp.

Scholtz M., van Hest B. y Schroeder W. (2003). Modelling of mercury emissions from background soils. Sci. Total Environ. 304 (1-3), 185-207.

DOI: 10.1016/S0048-9697(02)00568-5

Tarras-Wahlberg N.H., Flachier A., Lane S.N. y Sangfors O. (2001). Environmental impacts and metal exposure of aquatic ecosystems in rivers contaminated by small scale gold mining: The Puyango River basin, southern Ecuador. Sci. Total Environ. 278 (1), 239-261.

DOI: $10.1016 / \mathrm{S} 0048-9697(01) 00655-6$

Thangaradjou T., Subhashini P., Red S., Dilipan E. y Boyfriend E. (2014). Evidences for heavy metal contamination in surface sediments of seagrass ecosystem of Lakshadweep archipelago, India. Environ. Earth Sci. 71 (3), 1135-1146. DOI: 10.1007/s12665-013-2517-6
Velásquez-López P.C., Veiga M.M. y Hall K. (2010). Mercury balance in amalgamation in artisanal and small-scale gold mining: Identifying strategies for reducing environmental pollution in Portovelo-Zaruma, Ecuador. J. Cleaner Product. 18 (3), 226-232. DOI: 10.1016/j.jclepro.2009.10.010

Walsh G.E., Ainsworth K.A. y Rigby R. (1979). Resistance of red mangrove (Rhizophora mangle L.) seedlings to lead, cadmium, and mercury. Biotropica 11 (1), 22-27. DOI: $10.2307 / 2388167$

Wang'ondu V.W., Bosire J.O., Kairo J.G., Kinyamario J.I., Mwaura F.B., Dahdouh-Guebas F. y Koedam N. (2014). Litter fall dynamics of restored mangroves (Rhizophora mucronata Lamk. and Sonneratia alba Sm.) in Kenya. Rest. Ecology 22 (6), 824-831. DOI: $10.1111 / \mathrm{rec} .12149$

Zhang H., Feng X., Zhu J., Sapkota A., Meng B., Yao H., Qin H. y Larssen T. (2012). Selenium in soil inhibits mercury uptake and translocation in rice (Oryza sativa L.). Environ. Sci. Technol. 46 (18), 10040-10046. DOI: 10.1021/es302245r

Zhou Y., Peng Y., Li X. y Chen G. (2011). Accumulation and partitioning of heavy metals in mangrove rhizosphere sediments. Environ. Earth Sci. 64 (3), 799-807. DOI: $10.1007 / \mathrm{s} 12665-011-0904-4$ 